Pacific Journal of Mathematics

BRAID GROUP REPRESENTATIONS ARISING FROM TH 


\title{
BRAID GROUP REPRESENTATIONS ARISING FROM THE GENERALIZED CHIRAL POTTS MODELS
}

\author{
Etsuro Date, Michio Jimbo, Kei Miki*, and Tetsuji Miwa \\ Dedicated to Professor Heisuke Hironaka on his sixtieth birthday
}

A class of braid group representations are constructed for each non-singular bilinear form $B:(\mathbf{Z} / N Z)^{l} \times(\mathbf{Z} / N Z)^{l} \rightarrow \mathbf{Z} / N Z$ with $N$ odd. Associated link invariants are given as a Gauss sum involving the Seifert matrix and $B$. With a special choice of $B$ these representations are Yang-Baxterized to the $\operatorname{sl}(n)$ generalizations of the chiral Potts model discovered recently.

1. Introduction. The chiral Potts model [1-4] is a solvable lattice model whose Boltzmann weights are parametrized by a high genus curve and satisfy the star-triangle relation. The recent works [511] clarified the place occupied by this model in the updated catalogue of solvable lattice models. Like many other models the Boltzmann weights of the chiral Potts model constitute the $R$-matricesintertwiners of representations of the quantum group $U_{q}(\mathfrak{g})$. If $q$ is a root of unity and $\mathfrak{g}=\mathfrak{s l}(2)$ we get the chiral Potts model. In $[10,11]$ an $\mathfrak{s l}(n)$ generalization of the chiral Potts model has been obtained.

One of the most interesting features of solvable lattice models is its connection with the braid group representations. Given a solution to the Yang-Baxter equation, it is natural to ask the questions: Which representations of the braid groups arise therefrom and which invariants of links are obtained? The aim of this paper is to study the $\mathfrak{s l}(n)$ chiral Potts model from this point of view.

The trigonometric limit of the $\mathfrak{s l}(2)$ chiral Potts model has been known by Zamolodchikov and Fateev [12]. Kobayashi et al. [13] found that the braid group representations arising from it lead to link invariants related to the Seifert matrix. Goldschmidt and Jones [17] constructed a more general class of braid group representations by joining the Burau representation of the braid groups and the metaplectic representation of the symplectic groups over a finite field. They have also studied the corresponding link invariants and found the relation to the Alexander module and the Seifert matrix. In this paper

\footnotetext{
* Fellow of the Japan Society for the Promotion of Science for Japanese Junior Scientists.
} 
we generalize this construction to incorporate the $\mathfrak{s l}(n)$ chiral Potts models. Namely, we construct group homomorphisms from braid groups into certain finite dimensional $C^{*}$ algebras, and show that the braid group representations obtained from the generalized chiral Potts model fall into this class.

Fix a positive odd integer $N$ and a non-singular bilinear form $B:(\mathbf{Z} / N \mathbf{Z})^{l} \times(\mathbf{Z} / N \mathbf{Z})^{l} \rightarrow \mathbf{Z} / N \mathbf{Z}$. For each finite sequence $c$ of \pm 1 (which we call a configuration), we construct a $C^{*}$ algebra $\mathscr{A}(c)$ generated by unitary elements satisfying $q$ commutation relations, i.e., the relations of type $\alpha \beta=q^{m} \beta \alpha$ with $q$ a primitive $N$ th root of unity. Then we construct a functor $F$ from the category of uniform oriented tangles [15] to the category of vector spaces such that $F(c)=\mathscr{A}(c)$. In the special case $c=(1, \ldots, 1)$ this gives representations of the usual braid groups. The construction in [17] concerns this case with $l=1,2$. (Reading our first draft, Jones pointed out to us that the general case can also be handled through the Burau representation and the metaplectic characters.) The trace on $\mathscr{A}(c)$ enjoys the Markov property, so that we obtain invariants of uniform oriented tangles. These invariants are expressed as "Gauss sums" associated with the quadratic form $P \otimes B+{ }^{t} P \otimes{ }^{t} B$ where $P$ denotes a Seifert matrix of the link. In fact, they are invariants of the equivalence classes of Seifert matrices. For $N$ an odd prime, they can be evaluated in terms of classical Alexander polynomials provided the above quadratic form is non-singular. (See [17] in which a detailed study is given for $l=1,2$.)

In [14] Jones proposed the problem of Yang-Baxterization-to find solutions of the Yang-Baxter equation corresponding to given braid group representations. We show that with a particular choice of $B$ (and only in that case), the representations above can be YangBaxterized, and they are obtained from the $\mathfrak{s l}(n)$ chiral Potts model. $H$. Murakami taught us that this particular choice of $B$ is explained by the $n$-fold cyclic cover of $S^{3} \backslash L$ ( $L$ : link).

The intertwiner of the $\mathfrak{s l}(n)$ chiral Potts model splits into four pieces $[10,11]$

$$
R(\xi, \tilde{\xi})=S_{\tilde{r} r^{\prime}}^{-1} T_{r^{\prime} \tilde{r}^{\prime}} \bar{T}_{r \tilde{r}} S_{r \tilde{r}^{\prime}}
$$

We shall show that the operator $T$ (or $\bar{T}$ ) satisfies the Yang-Baxter equation independently of others, and more generally that $T, \bar{T}$ and $S$ satisfy the Yang-Baxter equation in a twisted sense (see (1.2) below). This gives an alternative proof of the Yang-Baxter equation for the $R$ 
matrix (1.1) [11]. Consider for simplicity the trigonometric limit. In the setting described above, the $T, \bar{T}$ and $S$ operators give rise to elements $T_{k}(c ; x) \in \operatorname{Hom}_{\mathbf{C}}\left(\mathscr{A}(c), \mathscr{A}\left(s_{k}(c)\right)\right) \quad(x \in \mathbf{C})$ for various configurations $c$. Here $s_{k}=(k, k+1)$ is a transposition acting on configurations. The twisted Yang-Baxter equation takes the form

$$
\begin{aligned}
& T_{k}\left(s_{k+1} s_{k}(c) ; x\right) T_{k+1}\left(s_{k}(c) ; x y\right) T_{k}(c ; y) \\
& \quad=T_{k+1}\left(s_{k} s_{k+1}(c) ; y\right) T_{k}\left(s_{k+1}(c) ; x y\right) T_{k+1}(c ; x) .
\end{aligned}
$$

The braiding operators $T_{k}(c)^{ \pm 1}$ obtained from $T_{k}(c ; x)$ in the limit of $x^{ \pm 1} \rightarrow 0$ coincide with those associated with a particular bilinear form of rank $l=n-1$.

The plan of our paper is as follows. In $\S 2$ we define the algebras $\mathscr{A}(c)$. In $\S 3$ we give the functors from the category of uniform oriented tangles to the category of vector spaces, and evaluate the resulting invariants. In $\S 4$ we extend a particular case of the braiding operators to the elementary operators of the $\mathfrak{s l}(n)$ chiral Potts models and prove the Yang-Baxter equations for them in $\mathscr{A}(c)$. Finally we show that the trigonometric Yang-Baxterization is possible only for this special case.

\section{Algebra $\mathscr{A}$.}

2.1. Notations. We fix a positive odd integer $N$, and a primitive $N$ th root of unity $q$. Let $L$ be a free $\mathbf{Z} / N Z$ module of rank $l$, and let $B$ be a non-singular bilinear form on $L$

$$
B: L \times L \rightarrow \mathbf{Z} / N \mathbf{Z}, \quad \operatorname{det} B \in(\mathbf{Z} / N \mathbf{Z})^{\times} .
$$

We denote by $A$ the skew-symmetric part of $B$

$$
A(a, b)=\frac{1}{2}(B(a, b)-B(b, a)) \text {. }
$$

Let - be a $\mathrm{Z} / N \mathrm{Z}$ linear isomorphism of $L$ such that

$$
B(a, \hat{b})=-B(b, a),
$$

and let $\vee$ be its inverse. Note that $B(a, b)=B(\hat{a}, \hat{b}), A(a, b)=$ $B\left(a, \frac{b+\hat{b}}{2}\right)$.

Let $M$ be a positive integer and let $\mathfrak{S}_{M}$ be the symmetric group of degree $M$, i.e., $w \in \mathfrak{S}_{M}$ is a bijective map from $\{1, \ldots, M\}$ to itself. We consider oriented strings. More precisely, a configuration is a map $c:\{1, \ldots, M\} \rightarrow\{ \pm 1\}$. We often write $(c(1), \ldots, c(M))$ to indicate a configuration $c$. We say that $c$ is of type $\left(M_{+}, M_{-}\right)$ where $M_{ \pm}=\#\{k \mid c(k)= \pm 1\}, M=M_{+}+M_{-}$. Graphically a configuration $c$ is a set of $M$ strings such that the $k$ th string (counted from 


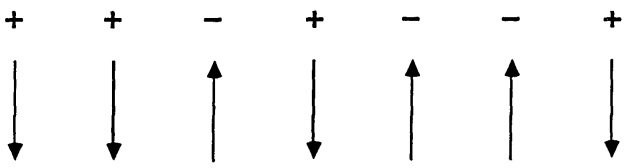

FIGURE 2.1

Configuration $(1,1,-1,1,-1,-1,1)$

left to right) is downward or upward according as $c(k)=1$ or -1 , respectively.

The symmetric group $\mathfrak{S}_{M}$ acts on the set of configurations of $M$ strings;

$$
w(c)=c \circ w^{-1} \text {. }
$$

Let $c, c^{\prime}$ be configurations of $M$ strings. There exists $w \in \mathfrak{S}_{M}$ such that $c^{\prime}=w(c)$ if and only if $c$ and $c^{\prime}$ are of the same type. We denote $c \sim c^{\prime}$ if this condition holds.

2.2. Algebra $\mathscr{A}(c)$. Let $c$ be a configuration of $M$ strings. We shall define a $\mathbf{C}$ algebra $\mathscr{A}(c)$ with a unit which we denote by 1 . If $M=0$ or $1, \mathscr{A}(c)$ is simply $\mathbf{C} \cdot 1$. If $M \geq 2$, we take generators $x_{k}^{a}=x_{k}^{a}(c)$ of $\mathscr{A}(c)$ where $1 \leq k \leq M-1$ and $a \in L$. We impose the following relations.

$$
\begin{aligned}
x_{k}^{0} & =1, \\
x_{k}^{a} x_{k}^{b} & =q^{A(b, a)} x_{k}^{a+b} \quad \text { if }(c(k), c(k+1))=(1,1) \\
& =q^{A(a, b)} x_{k}^{a+b} \quad \text { if }(c(k), c(k+1))=(-1,-1) \\
& =x_{k}^{a+b} \quad \text { if } c(k) \neq c(k+1), \\
x_{k}^{a} x_{k+1}^{b} & =q^{B(a, b)} x_{k+1}^{b} x_{k}^{a} \quad \text { if } c(k+1)=1 \\
& =q^{B(b, a)} x_{k+1}^{b} x_{k}^{a} \quad \text { if } c(k+1)=-1, \\
x_{k}^{a} x_{k^{\prime}}^{b} & =x_{k^{\prime}}^{b} x_{k}^{a} \quad \text { if }\left|k-k^{\prime}\right| \geq 2 .
\end{aligned}
$$

The set $\left\{x_{1}^{a_{1}} \cdots x_{M-1}^{a_{M-1}} \mid a_{k} \in L \quad(1 \leq k \leq M-1)\right\}$ constitutes a $\mathbf{C}$ linear basis of $\mathscr{A}(c)$. Therefore, if $M \neq 0, \operatorname{dim}_{\mathbf{C}} \mathscr{A}(c)=D^{M-1}$ where $D=N^{l}$.

We define a $\mathbf{C}$ linear map $\varepsilon: \mathscr{A}(c) \rightarrow \mathbf{C}$ by

$$
\begin{aligned}
\varepsilon\left(x_{1}^{a_{1}} \cdots x_{M-1}^{a_{M-1}}\right) & =1 & & \text { if } a_{1}=\cdots=a_{M-1}=0 \\
& =0 & & \text { otherwise, }
\end{aligned}
$$

and an anti-linear anti-involution $*: \mathscr{A}(c) \rightarrow \mathscr{A}(c)$ by

$$
\left(x_{k}^{a}\right)^{*}=x_{k}^{-a} \text {. }
$$


Then we have

$$
\varepsilon(a b)=\varepsilon(b a) \text { for } a, b \in \mathscr{A}(c),
$$

and $\mathscr{A}(c)$ equipped with the norm $|a|=\sup _{\lambda}\left\{|\lambda|^{1 / 2} \mid \lambda-a^{*} a\right.$ is not invertible $\}$ is a finite dimensional $C^{*}$-algebra.

2.3. Isomorphisms $l_{c^{\prime}}^{c}$. Suppose that $c$ and $c^{\prime}$ are configurations of $M$ strings such that $c \sim c^{\prime}$. We shall define canonical algebra isomorphisms

$$
l_{c^{\prime}}^{c}: \mathscr{A}(c) \rightarrow \mathscr{A}\left(c^{\prime}\right)
$$

such that

$$
\begin{array}{ll}
l_{c}^{c}=\mathrm{id}_{\mathscr{A}(c)}, & l_{c^{\prime \prime}}^{c^{\prime} \circ l_{c^{\prime}}^{c}=l_{c^{\prime \prime}}^{c},} \\
\varepsilon \circ l_{c^{\prime}}^{c}=\varepsilon, & * \circ l_{c^{\prime}}^{c}=l_{c^{\prime}}^{c} \circ * .
\end{array}
$$

We have in particular $l_{c}^{c^{\prime}} \circ l_{c^{\prime}}^{c}=\mathrm{id}_{\mathscr{A}(c)}$.

Suppose that $c^{\prime}=s_{k}(c)$ where $s_{k}$ is the transposition $(k, k+1)$. We shall define $l_{c^{\prime}}^{c}$ in this case as follows. If $c(k)=c(k+1)$, then $c=c^{\prime}$, and we set $l_{c^{\prime}}^{c}=\operatorname{id}_{\mathscr{A}(c)}$. Suppose that $c(k)=1$ and $c(k+1)=$ -1 . Then $c^{\prime}(k)=-1$ and $c^{\prime}(k+1)=1$. We define

$$
\begin{aligned}
& l_{c}^{c^{\prime}}\left(x_{k^{\prime}}^{a}\left(c^{\prime}\right)\right)=x_{k^{\prime}}^{a}(c) \quad \text { if }\left|k-k^{\prime}\right| \geq 2, \\
& \imath_{c}^{c^{\prime}}\left(x_{k-1}^{a}\left(c^{\prime}\right)\right)=x_{k-1}^{a}(c) x_{k}^{(a+\hat{a}) / 2}(c), \\
& l_{c}^{c^{\prime}}\left(x_{k}^{a}\left(c^{\prime}\right)\right)=x_{k}^{-\hat{a}}(c), \\
& l_{c}^{c^{\prime}}\left(x_{k+1}^{a}\left(c^{\prime}\right)\right)=x_{k+1}^{a}(c) x_{k}^{(a+\hat{a}) / 2}(c) .
\end{aligned}
$$

Note $\left[x_{k-1}^{a}(c), x_{k}^{(a+\hat{a}) / 2}(c)\right]=\left[x_{k+1}^{a}(c), x_{k}^{(a+\hat{a}) / 2}(c)\right]=0$, since $B(a, a+\hat{a})=0$. We also define

$$
\begin{aligned}
& l_{c^{\prime}}^{c}\left(x_{k^{\prime}}^{a}(c)\right)=x_{k^{\prime}}^{a}\left(c^{\prime}\right) \quad \text { if }\left|k-k^{\prime}\right| \geq 2, \\
& l_{c^{\prime}}^{c}\left(x_{k-1}^{a}(c)\right)=x_{k-1}^{a}\left(c^{\prime}\right) x_{k}^{(a+\check{a}) / 2}\left(c^{\prime}\right), \\
& l_{c^{\prime}}^{c}\left(x_{k}^{a}(c)\right)=x_{k}^{-\check{a}}\left(c^{\prime}\right), \\
& l_{c^{\prime}}^{c}\left(x_{k+1}^{a}(c)\right)=x_{k+1}^{a}\left(c^{\prime}\right) x_{k}^{(a+\check{a}) / 2}\left(c^{\prime}\right) .
\end{aligned}
$$

Proposition 2.1. $l_{c^{\prime}}^{c}$ and $l_{c}^{c^{\prime}}$ given by (2.4) and (2.5) define algebra homomorphisms. They are inverses to each other and compatible with $\varepsilon$ and *. 
Proof. The proof is by case checking. We show only one case $c(k)=$ $1, c(k+1)=-1$ in which we use (2.1):

$$
\begin{aligned}
& l_{c}^{c^{\prime}}\left(x_{k-1}^{a}\left(c^{\prime}\right)\right) l_{c}^{c^{\prime}}\left(x_{k-1}^{b}\left(c^{\prime}\right)\right) \\
& \quad=x_{k-1}^{a}(c) x_{k}^{(a+\hat{a}) / 2}(c) x_{k-1}^{b}(c) x_{k}^{(b+\hat{b}) / 2}(c) \\
& \quad=q^{-B(b,(a+\hat{a}) / 2)} x_{k-1}^{a}(c) x_{k-1}^{b}(c) x_{k}^{(a+\hat{a}+b+\hat{b}) / 2}(c) \\
& \quad=l_{c}^{c^{\prime}}\left(x_{k-1}^{a}\left(c^{\prime}\right) x_{k-1}^{b}\left(c^{\prime}\right)\right) .
\end{aligned}
$$

Now consider arbitrary $c$ and $c^{\prime}$ such that $c^{\prime}=w(c)$ for some $w \in \mathfrak{S}_{M}$. Choose a reduced decomposition of $w$

$$
w=s_{i_{1}} \cdots s_{i_{m}} .
$$

Define configurations $c^{(j)}(0 \leq j \leq m)$ by

$$
c^{(0)}=c, \quad c^{(j)}=s_{i_{m-j+1}}\left(c^{(j-1)}\right), \quad c^{(m)}=c^{\prime},
$$

and set

$$
l_{w}=l_{c^{(m)}}^{c^{(m-1)}} \circ \cdots \circ l_{c^{(2)}}^{c^{(1)}} \circ l_{c^{(1)}}^{c^{(0)}}
$$

Proposition 2.2. $l_{w}: \mathscr{A}(c) \rightarrow \mathscr{A}\left(c^{\prime}\right)$ is an algebra isomorphism defined independently of decompositions of $w$.

Proof. We need to show that the defining relations in $\mathfrak{S}_{M}$, i.e., $s_{k}^{2}=1, s_{k} s_{k+1} s_{k}=s_{k+1} s_{k} s_{k+1}, s_{k} s_{k^{\prime}}=s_{k^{\prime}} s_{k} \quad\left(\left|k-k^{\prime}\right| \geq 2\right)$, are preserved by $l_{c^{\prime}}^{c}$. For example, set $c^{(1)}=s_{k}(c)$ and $c^{(2)}=s_{k^{\prime}}(c)$. If $\left|k-k^{\prime}\right| \geq 2$, then we have to prove $l_{c^{\prime}}^{c(2)} l_{c^{(2)}}^{c}=l_{c^{\prime}}^{c^{(1)}} l_{c^{(1)}}^{c}$. The proofs of this and other necessary equalities are straightforward.

We define $l_{c^{\prime}}^{c}=l_{w}$. Then all the requirements of (2.3) are satisfied. The structure of the algebra $\mathscr{A}(c)$ is thus determined by the type $\left(M_{+}, M_{-}\right)$of the configuration $c$. We shall henceforth identify $\mathscr{A}(c)$ and $\mathscr{A}\left(c^{\prime}\right)$ with $c \sim c^{\prime}$ via the isomorphism $l_{c^{\prime}}^{c}$, and let them denoted by $\mathscr{A}=\mathscr{A}_{M_{+}, M_{-}}$.

2.4. Representation. Let us construct a representation of $\mathscr{A}(c)$. Suppose that $L$ is given by an exact sequence

$$
0 \rightarrow \operatorname{Ker} \pi \rightarrow(\mathbf{Z} / N \mathbf{Z})^{n} \stackrel{\pi}{\longrightarrow} L \rightarrow 0 .
$$

We identify a column vector $a={ }^{t}\left(a_{0}, \ldots, a_{n-1}\right) \bmod \operatorname{Ker} \pi$ with the element $\pi(a) \in L$. Set $(a, b)=\sum_{i=0}^{n-1} a_{i} b_{i}$. Suppose that $B$ is an $n \times n$ matrix with the matrix elements in $\mathbf{Z} / N \mathbf{Z}$ such that $(a, B b)=0$ if $a \in \operatorname{Ker} \pi$ or $b \in \operatorname{Ker} \pi$. Then a bilinear form $B(a, b)$ on $L$ is 
defined by $B(a, b)=(a, B b)=\left(b,{ }^{t} B a\right)$. Note that $B a,{ }^{t} B a$ and $A a$ belong to $(\operatorname{Ker} \pi)^{\perp}$. Set $V=\mathbf{C} v_{0} \oplus \cdots \oplus \mathbf{C} v_{N-1} \cong \mathbf{C}^{N}$. Let $Z$ and $X$ be the following linear operators on $V$,

$$
Z v_{i}=v_{i-1}, \quad X v_{i}=q^{i} v_{i},
$$

and let $Z_{i}$ and $X_{i}$ be the following operators acting on $V^{\otimes n}$,

$$
\begin{aligned}
& Z_{i}=1 \otimes \cdots \otimes \stackrel{i \text { th }}{Z} \otimes \cdots \otimes 1, \\
& X_{i}=1 \otimes \cdots \otimes \stackrel{i \text { th }}{X} \otimes \cdots \otimes 1 .
\end{aligned}
$$

For $a \in(\mathbf{Z} / N \mathbf{Z})^{n}$ we set

$$
Z^{a}=Z_{0}^{a_{0}} \cdots Z_{n-1}^{a_{n-1}}, \quad X^{a}=X_{0}^{a_{0}} \cdots X_{n-1}^{a_{n-1}} .
$$

Note that $Z^{a} X^{b}=q^{(a, b)} X^{b} Z^{a}$. Consider the subspace $W^{(0)}$ of $V^{\otimes n}$ given by

$$
W^{(0)}=\left\{w \in V^{\otimes n} \mid Z^{a} w=w \text { for } a \in \operatorname{Ker} \pi\right\} .
$$

If $a \in(\operatorname{Ker} \pi)^{\perp}$ then $X^{a}$ acts on $W^{(0)}$. With all these preparations we can construct a representation $\rho$ of $\mathscr{A}(c)$ on $\left(W^{(0)}\right)^{\otimes M}$ as follows.

$$
\rho\left(x_{k}^{a}(c)\right)=1 \otimes \cdots \otimes g_{c(k)}^{k_{\text {th }}^{a}} \otimes h_{c(k+1)}^{a+1) \text { th }} \otimes \cdots \otimes 1
$$

where

$$
\begin{array}{ll}
g_{+}^{a}=Z^{-a / 2} X^{B a}, & g_{-}^{a}=Z^{-a / 2} X^{t} B a \\
h_{+}^{a}=Z^{a / 2} X^{t} B a, & h_{-}^{a}=Z^{a / 2} X^{B a} .
\end{array}
$$

2.5. Locality. Fix a type $\left(M_{+}, M_{-}\right)$of configurations. For configurations $c, c^{\prime}$ of the same type, the expression of $x_{k}^{a}(c)$ in terms of $x_{k^{\prime}}^{a}\left(c^{\prime}\right)$ is in general nonlocal, i.e., depends on the latter with $\left|k^{\prime}-k\right|>$ 1. Nevertheless the left regular representation on $\mathscr{A}=\mathscr{A}_{M_{+}}, M_{-}$

$$
\mathscr{M}(\alpha) \beta=\alpha \beta \quad(\alpha, \beta \in \mathscr{A})
$$

can be made local in the sense explained below.

For each $c$ let us introduce the basis elements

$$
y(c)^{a_{1} \cdots a_{M-1}}=q^{-\frac{1}{2} \Sigma_{k=1}^{M-2} B^{c(k+1)}\left(a_{k}, a_{k+1}\right)} x_{1}^{a_{1}}(c) \cdots x_{M-1}^{a_{M-1}}(c),
$$

where $a_{k} \in L$ and

$$
B^{+1}(a, b)=B(a, b), \quad B^{-1}(a, b)=B(b, a) .
$$

We define the isomorphism $\rho_{c}: \mathscr{A} \simeq\left(C^{D}\right)^{\otimes(M-1)}$ by

$$
\rho_{c}\left(y(c)^{a_{1}, \ldots, a_{M-1}}\right)=v_{a_{1}} \otimes \cdots \otimes v_{a_{M-1}},
$$

where $\left\{v_{a}\right\}_{a \in L}$ signifies the standard basis of $\mathbf{C}^{D}$. 
Let $c, c^{\prime}$ be two configurations of the type $\left(M_{+}, M_{-}\right)$. We set for $\alpha \in \mathscr{A}$

$$
\begin{aligned}
\rho_{c^{\prime}} \circ & \mathscr{M}(\alpha) \circ \rho_{c}^{-1}\left(v_{a_{1}} \otimes \cdots \otimes v_{a_{M-1}}\right) \\
& =\sum_{a_{1}^{\prime} \cdots a_{M-1}^{\prime}}(\alpha)_{c^{\prime} a_{1}^{\prime} \cdots a_{M-1}^{\prime}}^{c a_{1-1}^{\prime}} v_{a_{1}^{\prime}} \otimes \cdots \otimes v_{a_{M-1}^{\prime}} .
\end{aligned}
$$

Clearly one has

$$
(\alpha \beta)_{c^{\prime \prime} a_{1}^{\prime \prime} \cdots a_{M-1}^{\prime \prime}}^{c a_{1} \cdots a_{M-1}}=\sum_{a_{1}^{\prime}, \ldots, a_{M-1}^{\prime}}(\alpha)_{c^{\prime \prime} a_{1}^{\prime \prime} \cdots a_{M-1}^{\prime \prime}}^{c^{\prime} a^{\prime} \cdot a^{\prime}}(\beta)_{c^{\prime} a_{1}^{\prime} \cdots a_{M-1}^{\prime}}^{c a_{1} \cdots a_{M-1}} .
$$

We say that $\alpha \in \mathscr{A}$ is local relative to the pair $\left(c, c^{\prime}\right)$ if the matrix element $(\alpha)_{c^{\prime} a_{1}^{\prime \cdots} \cdots a_{M-1}^{\prime}}^{c a_{1} \cdots a_{M-1}}$ has the form

$$
\left(\prod_{l \neq k} \delta_{a_{l} a_{l}^{\prime}}\right) w\left(a_{k-1}, a_{k}, a_{k+1}, a_{k}^{\prime}\right)
$$

for some function $w(a, b, e, f)$.

The elements $\left(x_{k}^{b}(c)\right)$ are local relative to $\left(c, s_{k}(c)\right)$; in fact

$$
\begin{array}{r}
\left(x_{k}^{b}(c)\right)_{s_{k}(c) a_{1}^{\prime} \cdots a_{M-1}^{\prime}}^{c a_{1} \cdots a_{M-1}}=\left(\prod_{l \neq k} \delta_{a_{l} a_{l}^{\prime}}\right) \delta_{a_{k}+b a_{k}^{\prime}} \omega\left(a_{k-1}, a_{k}, a_{k+1}, a_{k}^{\prime}\right) \\
\quad \text { for } c(k)=c(k+1)=1, \\
=\left(\prod_{l \neq k} \delta_{a_{l} a_{l}^{\prime}}\right) \delta_{a_{k}+b a_{k}^{\prime}} \omega\left(a_{k+1}, a_{k}^{\prime}, a_{k-1}, a_{k}\right) \\
\quad \text { for } c(k)=c(k+1)=-1, \\
=\left(\prod_{l \neq k} \delta_{a_{l} a_{l}^{\prime}}\right) \delta_{\bar{a}_{k}-a_{k}-b \hat{a}_{k}^{\prime}} \omega\left(a_{k}^{\prime}, a_{k-1}, a_{k}, a_{k+1}\right)^{-1} \\
\quad \begin{array}{r}
\text { for } c(k)=1, c(k+1)=-1, \\
\delta_{\bar{a}_{k}-\hat{a}_{k}-\hat{b} a_{k}^{\prime}} \omega\left(a_{k}, a_{k+1}, a_{k}^{\prime}, a_{k-1}\right)^{-1} \\
\text { for } c(k)=-1, c(k+1)=1,
\end{array}
\end{array}
$$

where

$$
\begin{aligned}
\omega(a, b, e, f) & =q^{B(f-b, \hat{a}+e) / 2+A(b, f)} \quad \text { for } a, b, e, f \in L, \\
\bar{a}_{k} & =\left(a_{k-1}+\hat{a}_{k-1}+a_{k+1}+\hat{a}_{k+1}\right) / 2 .
\end{aligned}
$$

The matrix elements of $x_{k}^{b}(c)$ are also local relative to $(c, c)$. 


\section{Link invariants.}

3.1. Elements $T_{k}(c), E_{k}(c)$. Set

$$
f(a)=\frac{1}{\sqrt{D}} q^{B(a, a) / 2}, \quad \tilde{f}(a)=\frac{1}{\sqrt{D}} \sum_{b} f(b) q^{B(a, b)}, \quad D=N^{l}
$$

Note that $g(\hat{a})=g(a)=g(-a)$ for $g=f$ or $\tilde{f}$. For each configuration $c=(c(1), \ldots, c(M))$, we define the following elements in $\mathscr{A}(c)$ :

$$
\begin{aligned}
T_{k}(c) & =\sum_{a} f(a)^{*} x_{k}^{a}(c) \quad \text { if } c(k)=c(k+1), \\
& =\sum_{a} \tilde{f}(a) x_{k}^{a}(c) \quad \text { if } c(k) \neq c(k+1), \\
E_{k}(c) & =\frac{1}{\sqrt{D}} \sum_{a} x_{k}^{a}(c) \quad \text { if } c(k) \neq c(k+1),
\end{aligned}
$$

where $k=1, \ldots, M-1$ and $f(a)^{*}$ means the complex conjugate. Here and in what follows, whenever $E_{k}(c)$ appears the condition $c(k) \neq c(k+1)$ is implied. Clearly, if $\left|k-k^{\prime}\right|>1$ then

$$
\begin{aligned}
& T_{k}\left(s_{k^{\prime}}(c)\right) T_{k^{\prime}}(c)=T_{k^{\prime}}\left(s_{k}(c)\right) T_{k}(c) \\
& E_{k}\left(s_{k^{\prime}}(c)\right) T_{k^{\prime}}(c)=T_{k^{\prime}}(c) E_{k}(c), \quad\left[E_{k}(c), E_{k^{\prime}}(c)\right]=0
\end{aligned}
$$

Using $\sum_{b} q^{B(a, b)}=D \delta_{a 0}$ it is easy to check that

$$
\begin{gathered}
T_{k}(c)^{*}=T_{k}(c)^{-1}, \quad T_{k}\left(s_{k}(c)\right)=T_{k}(c) \\
E_{k}(c)^{*}=E_{k}(c), \quad E_{k}\left(s_{k}(c)\right)=E_{k}(c) \\
E_{k}(c) x_{k}^{a}(c)=E_{k}(c)=x_{k}^{a}(c) E_{k}(c) \\
E_{k}(c) x_{k \pm 1}^{a}(c) E_{k}(c)=\delta_{a 0} \sqrt{D} E_{k}(c)
\end{gathered}
$$

3.2. Uniform oriented tangles. Let $\mathscr{T}$ denote the category of uniform oriented tangles in the sense of [15]. (Recall that an $(M, M)$ tangle $t$ is called uniform if the drawing of $t$ intersects each horizontal line between the top edge and the bottom edge at exactly $M, M-1$ or $M-2$ points; see Fig. 3.1.) The objects of $\mathscr{T}$ are configurations in the sense of $\S 2$. If $c, c^{\prime}$ are configurations of the same type, we let $\operatorname{hom}\left(c, c^{\prime}\right)$ be the set of uniform oriented tangles with $c$ on the top and $c^{\prime}$ on the bottom; we set $\operatorname{hom}\left(c, c^{\prime}\right)=\varnothing$ otherwise. 



t'ot

$t^{\prime}$

FIGURE 3.1

Uniform oriented tangles

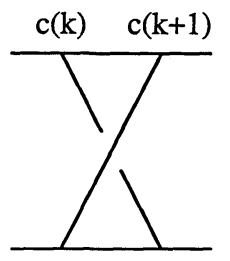

$\sigma_{\mathrm{k}}^{+}(\mathrm{c})$

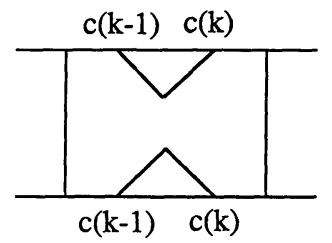

$\theta_{\mathrm{k}}^{+}(\mathrm{c})$



$\overline{\sigma_{k}^{-}}(c)$

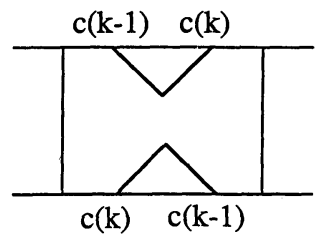

$\overline{\theta_{\mathrm{k}}}(\mathrm{c})$

FIGURE 3.2

Elementary tangles

Given two tangles $t \in \operatorname{hom}\left(c, c^{\prime}\right), t^{\prime} \in \operatorname{hom}\left(c^{\prime}, c^{\prime \prime}\right)$ their composition $t^{\prime} \circ t \in \operatorname{hom}\left(c, c^{\prime \prime}\right)$ is defined by concatenation as in Fig. 3.1.

We shall define a functor $\boldsymbol{F}$ from $\mathscr{T}$ to the category of $\mathbf{C}$ vector spaces. Let $\sigma_{k}^{ \pm}(c), \theta_{k}^{ \pm}(c)$ be the elementary tangles shown in Fig. 3.2. Any uniform oriented tangle can be written as a composition of them. 
The functor $F$ is specified as follows.

(i) $F(c)=\mathscr{A}(c)$ for a configuration $c$,

(ii) $F\left(\sigma_{k}^{ \pm}(c)\right)=\mathscr{M}\left(T_{k}(c)^{ \pm 1}\right), F\left(\theta_{k}^{ \pm}(c)\right)=\mathscr{M}\left(E_{k}(c)\right)$.

Here $\mathscr{M}(x) \in \operatorname{End}(\mathscr{A})$ denotes the left multiplication by $x \in \mathscr{A}$. We shall verify the well-definedness of $F$ in $\S 3.3$.

Let $\operatorname{tr}$ denote the usual matrix trace on $\operatorname{End}(\mathscr{A})$ so normalized that $\operatorname{tr}(1)=1$. Note that $\operatorname{tr}(\mathscr{M}(\alpha))=\varepsilon(\alpha)$ for $\alpha \in \mathscr{A}$.

Proposition 3.1. The following defines an invariant of oriented links:

$$
\tau(t)=D^{(M-1) / 2} \operatorname{tr}(F(t)), \quad t \in \operatorname{hom}(c, c)
$$

where $c$ is a configuration of $M$ strings.

The proof will be given in $\S 3.3$.

REMARK. The matrix elements (in the left regular representation) of $T_{k}(c)^{ \pm 1}$ relative to $\left(c, s_{k}(c)\right)$ and of $E_{k}(c)$ relative to $(c, c)$ or $\left(c, s_{k}(c)\right)$ are local in the sense of $\S 2.5$.

3.3. Well-definedness of $F, \tau$. First note that $(3.3 \mathrm{a}, \mathrm{b})$ imply

$$
F\left(t^{T}\right)=F(t)^{*} \quad \text { for } t \in \operatorname{hom}\left(c, c^{\prime}\right),
$$

where $t^{T} \in \operatorname{hom}\left(c^{\prime}, c\right)$ denotes the tangle obtained by the vertical flip with respect to the middle horizontal line [15], and $*$ means the adjoint with respect to the hermitian form $(\alpha, \beta)=\varepsilon\left(\alpha \beta^{*}\right)$ on $\mathscr{A}(c)$.

To prove the well-definedness of $F$ we are to check the relations corresponding to the Reidemeister moves R1-R10 as shown in Fig. 3.3.

The cases R1-4 and R5 correspond to (3.2) and (3.3a) respectively. In view of (3.5), it remains to verify the following relations.

$$
\begin{gathered}
T_{k}(c)^{ \pm 1} E_{k}(c)=E_{k}(c), \\
T_{k}\left(s_{k+1} s_{k}(c)\right) T_{k+1}\left(s_{k}(c)\right) T_{k}(c) \\
=T_{k+1}\left(s_{k} s_{k+1}(c)\right) T_{k}\left(s_{k+1}(c)\right) T_{k+1}(c), \\
E_{k}\left(c^{\prime}\right) E_{k \pm 1}\left(c^{\prime}\right) E_{k}(c)=E_{k}(c),
\end{gathered}
$$

In (3.6c) $c^{\prime}$ denotes the configuration such that $c^{\prime}(k-1)=-c^{\prime}(k)=$ $c^{\prime}(k+1)=c(k \pm 1), c^{\prime}(j)=c(j)(j \neq k-1, k, k+1)$. In (3.6d) $\varepsilon= \pm 1$ and $c^{\prime \prime}=s_{k}(c)$ if $c(k) \neq c(k+1),=s_{k \pm 1} s_{k}(c)$ otherwise. Among these, (3.6a) and (3.6c) are direct consequences of (3.3c) and (3.3d), respectively. 
R1

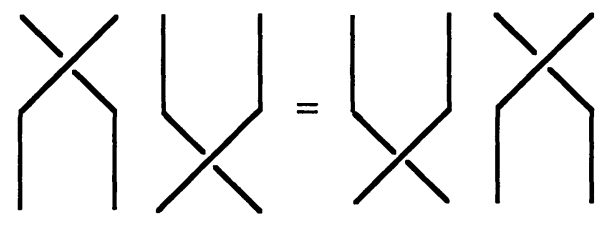

R2

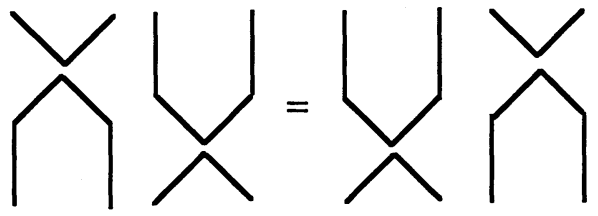

$\mathrm{R} 3$

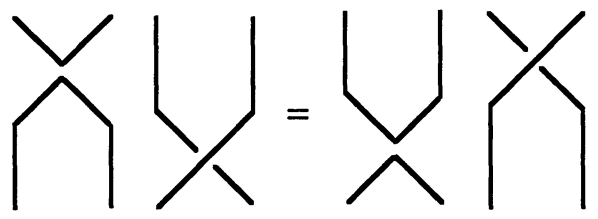

R4

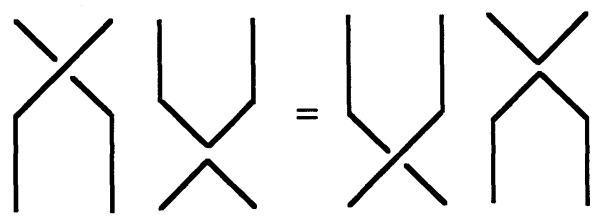

R5
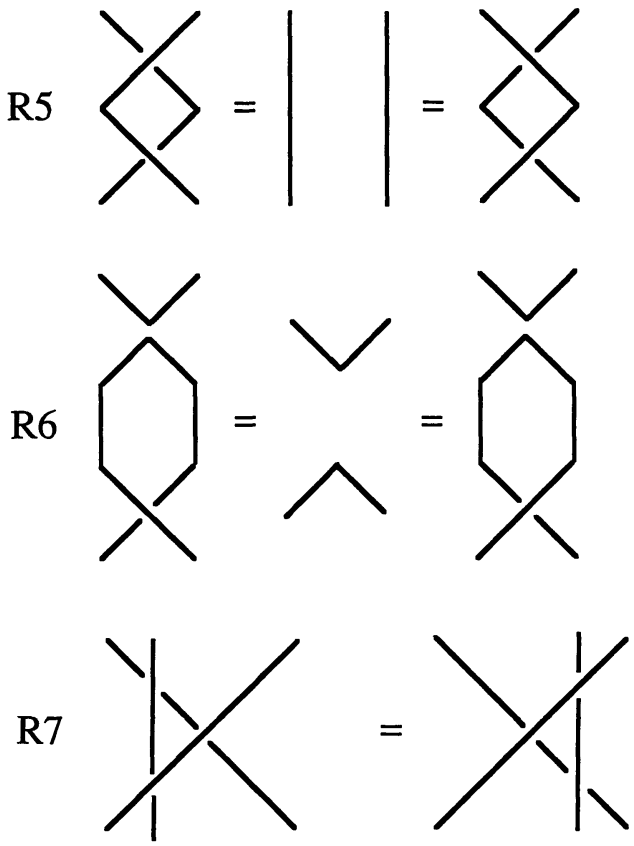

Figure 3.3 (continues) 

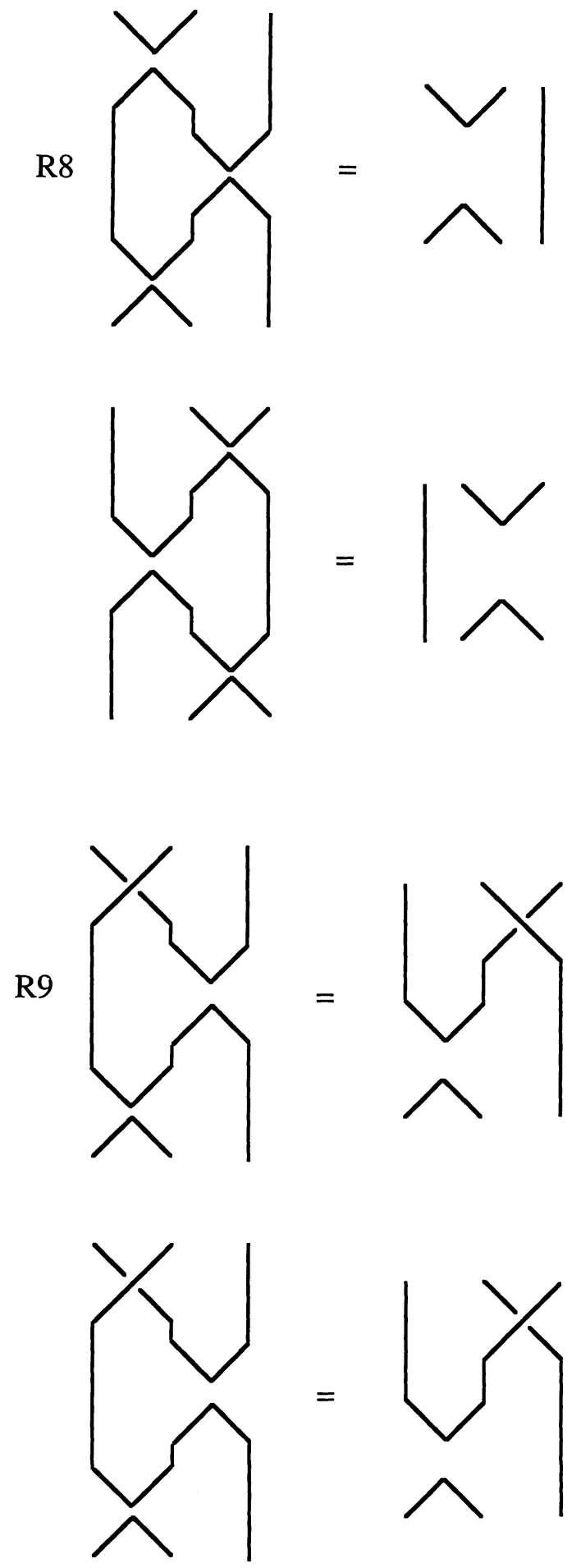

Figure 3.3 (continues) 


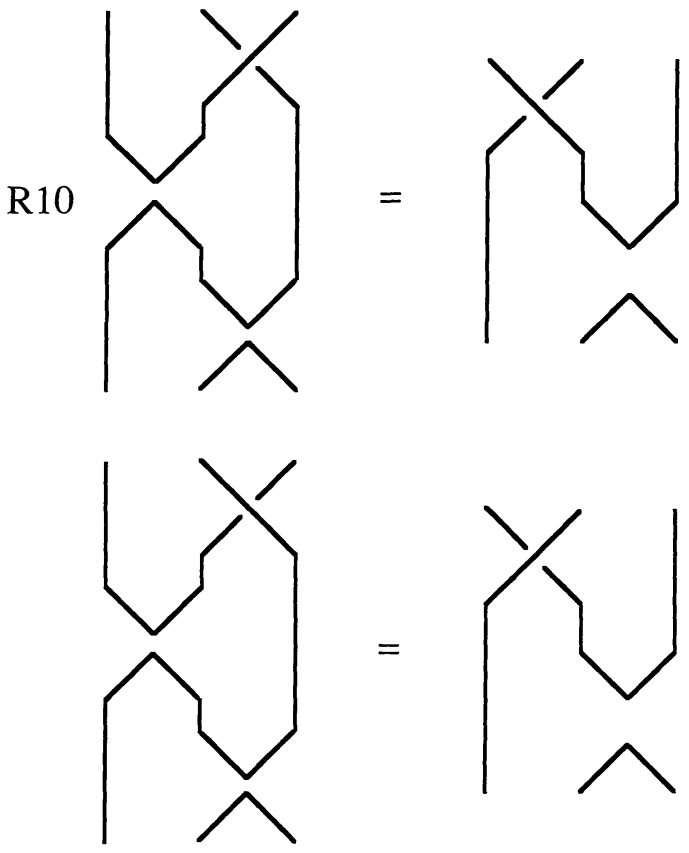

Figure 3.3

Reidemeister moves. Figures obtained by vertical flip are omitted. The strings should be oriented in all possible ways

Proof of (3.6b). There are altogether 8 cases according to the choices of $c(k), c(k+1), c(k+2)$. As an illustration we shall prove the case $(c(k), c(k+1), c(k+2))=(1,-1,1)$. In this case $(3.6 \mathrm{~b})$ reads

$$
\begin{aligned}
& \sum_{a, b, d} \tilde{f}(a) x_{k}^{-\hat{a}}(c) f(b)^{*} x_{k+1}^{b}(c) x_{k}^{(b+\hat{b}) / 2}(c) \tilde{f}(d) x_{k}^{d}(c) \\
& \quad=\sum_{a, b, d} \tilde{f}(a) x_{k+1}^{-\check{a}}(c) f(b)^{*} x_{k}^{b}(c) x_{k+1}^{(b+\check{b}) / 2}(c) \tilde{f}(d) x_{k+1}^{d}(c) .
\end{aligned}
$$

Equating the coefficients of $x_{k}^{a}(c) x_{k+1}^{b}(c)$ in both sides, we are to show

$$
\begin{aligned}
& \sum_{d} \tilde{f}(d) f(b)^{*} \tilde{f}(a+\hat{d}-(b+\hat{b}) / 2) q^{-B(b, a+\hat{d})} \\
& \quad=\sum_{d} \tilde{f}(d) f(a)^{*} \tilde{f}(b+\check{d}-(a+\check{a}) / 2) q^{B(\check{d}, a)} .
\end{aligned}
$$

Summing over $d$ we find that the left-hand side becomes

$$
\begin{gathered}
\sum_{e} f(e) f(b)^{*} f(b+e) q^{B(e, a)+B((b+\hat{b}) / 2, e)} \\
=D^{-3 / 2} \sum_{e} q^{B(e+b, e+a)-B(b, a)}
\end{gathered}
$$






FIGURE 3.4

Markov property

Likewise the right-hand side becomes

$$
\begin{gathered}
\sum_{e} f(e) f(a)^{*} f(a-e) q^{-B(b, a+e)+B((a+\check{a}) / 2, e)} \\
=D^{-3 / 2} \sum_{e} q^{B(e-b+a, e)-B(b, a)} .
\end{gathered}
$$

Our assertion follows from this.

Corollary 3.2. Suppose $c(k)=1(\forall k)$. Then the $\left\{T_{k}(c)\right\}$ provide a homomorphism from the $M$ string braid group into an algebra $\mathscr{A}(c)$.

Proof of (3.6d) can be done in a similar manner, so we omit it.

Proof of Proposition 3.1. We are to verify the invariance of $\tau$ under the Markov moves, namely (Fig. 3.4)

$$
\begin{array}{ll}
\tau\left(t \circ t^{\prime}\right)=\tau\left(t^{\prime} \circ t\right), & t \in \operatorname{hom}\left(c, c^{\prime}\right), t^{\prime} \in \operatorname{hom}\left(c^{\prime}, c\right), \\
\tau\left(t \circ \theta_{M}^{+}\right)=\tau(t), & t \in \operatorname{hom}(c, c),
\end{array}
$$

where $c, c^{\prime}$ are configurations of $M$ strings. The proof is straightforward.

3.4. Link invariants and Alexander polynomials. In this section we restrict ourselves to the configurations $c$ such that $c(k)=1$ for all $k$. In this case the uniform tangles are nothing but the usual braids. Let $t$ be a braid on $M$ strings with $v$ crossings, and let $K=v-M+1$.

Proposition 3.3. Let $P=\left(p_{i j}\right)_{1 \leq i, j \leq K}$ denote a Seifert matrix for the closure of $t$. Then $\tau(t)$ is given by

$$
\tau(t)=D^{-K / 2} \sum_{a_{1}, \ldots, a_{K}} q^{Q}, \quad Q=\sum_{1 \leq i, j \leq K} p_{i j} B\left(a_{i}, a_{j}\right) .
$$

Proof. Fix a drawing of the braid, and let $(k, i), 1 \leq i \leq m_{k}$, denote the $i$ th crossing (counted from top to bottom) between the 
$k$ th and $(k+1)$ th column. The sign of the crossing $(k, i)$ is denoted by $\varepsilon_{i}^{(k)}= \pm 1$. We write $(k, i)<\left(k^{\prime}, i^{\prime}\right)$ if the crossing $(k, i)$ is above $\left(k^{\prime}, i^{\prime}\right)$ in the drawing. In these notations we have

$$
\begin{aligned}
\tau(t)= & D^{-K / 2} \sum q^{Q}, \\
Q= & \sum \frac{1-\varepsilon_{i}^{(k)}}{2} B\left(a_{i}^{(k)}, a_{i}^{(k)}\right) \\
& +\sum_{(k, i)<(k, j)} B\left(a_{i}^{(k)}, a_{j}^{(k)}\right)-\sum_{(k, i)<(k+1, j)} B\left(a_{i}^{(k)}, a_{j}^{(k+1)}\right),
\end{aligned}
$$

where the sum ranges over $a_{i}^{(k)} \in L$ satisfying

$$
\sum a_{i}^{(k)}=0 \text { for } k=1, \ldots, M-1 .
$$

The substitution $b_{i}^{(k)}=\sum_{j=1}^{i} a_{j}^{(k)} \quad\left(1 \leq i \leq m_{k}-1\right)$ brings $Q$ into the form

$$
\begin{aligned}
Q= & -\sum \frac{\varepsilon_{i}^{(k)}+\varepsilon_{i+1}^{(k)}}{2} B\left(b_{i}^{(k)}, b_{i}^{(k)}\right) \\
& +\sum\left(\frac{1+\varepsilon_{i+1}^{(k)}}{2} B\left(b_{i}^{(k)}, b_{i+1}^{(k)}\right)+\frac{-1+\varepsilon_{i+1}^{(k)}}{2} B\left(b_{i+1}^{(k)}, b_{i}^{(k)}\right)\right) \\
& +\sum\left(B\left(b_{i}^{(k)}, b_{i^{*}}^{(k+1)}\right)-B\left(b_{i-1}^{(k)}, b_{i^{*}}^{(k+1)}\right)\right) .
\end{aligned}
$$

In the sum, $i^{*}$ signifies the largest $j$ such that $(k+1, j)<(k, i)$.

Next we draw a Seifert surface for $t$ using Seifert's algorithm (see e.g. [16]). Let $\gamma_{i}^{(k)}$ be the cycle passing counterclockwise through $(k, i)$ and $(k, i+1)$ (Fig. 3.5).

On these cycles the nonzero values of the Seifert form $\varphi$ are given by

$$
\begin{aligned}
& \varphi\left(\gamma_{i}^{(k)}, \gamma_{i}^{(k)}\right)=-\frac{\varepsilon_{i}^{(k)}+\varepsilon_{i+1}^{(k)}}{2}, \\
& \varphi\left(\gamma_{i}^{(k)}, \gamma_{i+1}^{(k)}\right)=\frac{1+\varepsilon_{i+1}^{(k)}}{2}, \quad \varphi\left(\gamma_{i+1}^{(k)}, \gamma_{i}^{(k)}\right)=\frac{-1+\varepsilon_{i+1}^{(k)}}{2}, \\
& \varphi\left(\gamma_{i}^{(k)}, \gamma_{j}^{(k+1)}\right)=1 \\
& \quad \text { if }(j, k+1)<(i, k)<(j+1, k+1)<(i+1, k), \\
& \varphi\left(\gamma_{i}^{(k)}, \gamma_{j}^{(k+1)}\right)=-1 \\
& \quad \text { if }(i, k)<(j, k+1)<(i+1, k)<(j+1, k+1) .
\end{aligned}
$$

Our assertion follows from this. 


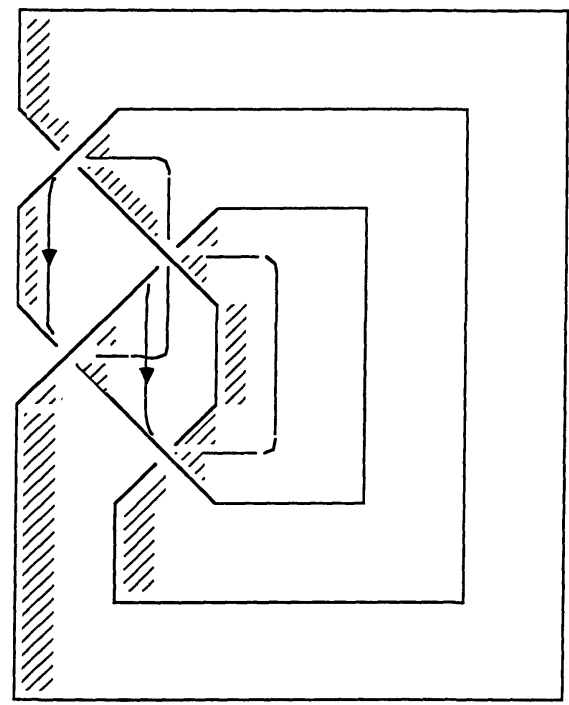

FIGURE 3.5

Seifert matrix

Remark. Fix $B$. The quantity (3.7) determined by a Seifert matrix $P$ is in fact an invariant of the equivalence classes of the Seifert matrices ([18], [19]).

Assume now that $N=p$ is an odd prime. For $k \not \equiv 0 \bmod p$ let

$$
s(k, p)=\sum_{j=0}^{p-1} q^{j^{2}}, \quad q=\exp \left(\frac{2 \pi \sqrt{-1} k}{p}\right)
$$

denote the Gauss sum. The following is well known.

Lemma 3.4. (1) Denoting by $\left(\frac{k}{p}\right)$ the Legendre symbol, we have

$$
s(k, p)=\sqrt{p}\left(\frac{k}{p}\right) .
$$

(2) Let $\phi(a)$ be a nondegenerate quadratic form of rank $l$ on the finite field $\mathbf{F}_{p}$ with $p$ elements. Then

$$
\sum_{a \in F_{p}^{l}} q^{\phi(a)}=s(k, p)^{l-1} s(k \operatorname{det} \phi, p) .
$$

Returning to the setting of Proposition 3.3, define

$$
\Delta(x)=\operatorname{det}\left(P-x^{t} P\right) .
$$


Then $x^{-K / 2} \Delta(x)$ is the classical Alexander polynomial. Upon symmetrizing $Q$ the determinant of the quadratic form in the Gauss sum (3.7) is given by

$$
\begin{aligned}
\operatorname{det}\left(P \otimes B+{ }^{t} P \otimes{ }^{t} B\right) & =(\operatorname{det} B)^{K} \operatorname{det}\left(P \otimes 1+{ }^{t} P \otimes B^{-1 t} B\right) \\
& =(\operatorname{det} B)^{K} \prod_{i} \Delta\left(x_{i}\right),
\end{aligned}
$$

where $x_{i}$ ranges over the eigenvalues of the matrix $-B^{-1 t} B$. Hence, if this is nonzero, the invariant $\tau(t)$ is written in terms of the Alexander polynomial.

REMARK. Given a link $L$ in $S^{3}$ consider the $n$-fold cyclic cover $M_{n}$ of $S^{3}$ branched along $L$. It is known that the first homology group of $M_{n}$ can be represented as

$$
H_{1}\left(M_{n}\right)=\mathbf{Z}^{K} \otimes \mathbf{Z}^{n-1} / Q \mathbf{Z}^{K} \otimes \mathbf{Z}^{n-1}, \quad Q=P \otimes B+{ }^{t} P \otimes{ }^{t} B,
$$

where $P$ is a Seifert matrix of $L$ of size $K$, and $B=\left(B_{i j}\right)$ is the matrix given by $B_{i j}=1$ for $1 \leq i \leq j \leq n-1$ and $B_{i j}=0$ otherwise. The bilinear form associated with $B$ is the one relevant to the $\widehat{\mathfrak{s l}}(n)$ chiral Potts model (see $\S 4$ ). We are indebted to H. Murakami about this comment.

4. Yang-Baxterization. Except in $\S 4.3$ we shall henceforth deal with the following case

$$
\begin{gathered}
0 \rightarrow(\mathbf{Z} / N \mathbf{Z})^{t}(1, \ldots, 1) \rightarrow(\mathbf{Z} / N \mathbf{Z})^{n} \stackrel{\pi}{\rightarrow} L \rightarrow 0 \\
A(a, b)=\sum_{i}\left(a_{i} b_{i+1}-a_{i+1} b_{i}\right), \quad B(a, b)=-2 \sum_{i} a_{i}\left(b_{i}-b_{i+1}\right) .
\end{gathered}
$$

We set

$$
\nu_{i}={ }^{t}(0, \ldots, \stackrel{i \text { th }}{1}, \ldots, 0) .
$$

We note that $\hat{\nu}_{i}=\nu_{i+1}$.

4.1. The operator $T$. Our goal is to show that the twisted braid relations (3.6b) can be Yang-Baxterized in this case (Theorem 4.1, Corollary 4.2). To state the result we need to prepare some notations. Fix $\gamma=\left(\lambda_{i}, \mu_{i}\right)_{0 \leq i<n} \in\left(\mathbf{C}^{\times}\right)^{2 n}$ such that $\lambda_{i} \neq \mu_{j} \quad(\forall i, j)$, and consider an algebraic curve [11]

$$
C_{\gamma}=\left\{r=\left(u_{i}, v_{i}\right)_{0 \leq i<n} \in\left(\mathbf{C}^{\times}\right)^{2 n} \mid u_{i}^{N}+\lambda_{i}=v_{j}^{N}+\mu_{j}(0 \leq i, j<n)\right\} .
$$

Denote by $\dagger$ the automorphism of the curve such that

$$
r^{\dagger}=\left(q u_{i}, v_{i}\right)_{0 \leq i<n} \text { for } r=\left(u_{i}, v_{i}\right)_{0 \leq i<n} .
$$


We define the functions $\sigma_{r \tilde{r}}^{\alpha}(a)\left(r, \tilde{r} \in C_{\gamma}, a \in L, \alpha \in \mathbf{Z} / n \mathbf{Z}\right)$ by the recurrence relations

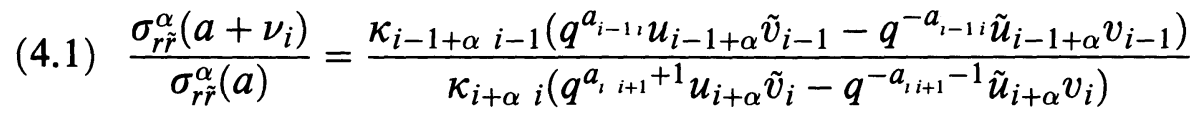

for all $i=0,1, \ldots, n-1$, and the normalization condition $\sigma_{r \tilde{r}}^{\alpha}(0)=$ 1. Here

$$
\kappa_{i j}^{N}=\frac{1}{\lambda_{i}-\mu_{j}}, \quad a_{i j}=a_{i}-a_{j},
$$

and the suffixes $i, j$ should be read modulo $n$. These functions satisfy the following relations:

$$
\sigma_{r^{\dagger} \tilde{r}^{\dagger}}^{\alpha}(a)=\sigma_{r \tilde{r}}^{\alpha}(a), \quad \sigma_{r \tilde{r}^{\dagger}}^{\alpha}(a)=\sigma_{\tilde{r} r^{\dagger}}^{\alpha}(-a)^{-1} .
$$

Let us denote the Fourier transform of $\sigma_{r \tilde{r}^{\dagger}}^{\alpha}$ by

$$
\phi_{\tilde{r} r}^{\alpha}(a)=\frac{1}{\sqrt{D}} \sum_{b} \sigma_{r \tilde{r}^{\dagger}}^{\alpha}(b) q^{-B(b, a)} .
$$

Let us extend the notion of a configuration by assigning to each string a triple $(r, c, \alpha)$ consisting of $r \in C_{\gamma}, c= \pm 1$ and $\alpha \in \mathbf{Z} / n \mathbf{Z}$. As before $c= \pm 1$ signifies that the string is going downwards or upwards respectively. We define a decorated configuration $d$ of $M$ strings to be a map

$$
\begin{gathered}
d:\{1, \ldots, M\} \rightarrow C_{\gamma} \times\{ \pm 1\} \times \mathbf{Z} / n \mathbf{Z}, \\
k \mapsto d(k)=(r(k), c(k), \alpha(k))
\end{gathered}
$$

where $r(k)=\left(u(k)_{i}, v(k)_{i}\right)_{0 \leq i<n}$ and the $\alpha(k)$ are such that

$$
\alpha(k)-\alpha(k+1)=\frac{c(k)+c(k+1)}{2} .
$$
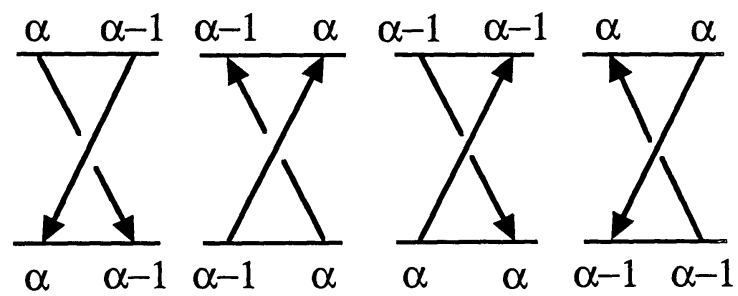

FIGURE 4.1

Change of $\alpha$ in the decorated configuration 
We shall write $\mathscr{A}(d), x_{k}^{a}(d)$ for $\mathscr{A}(c), x_{k}^{a}(c)$ respectively. Let us define the action of symmetric group $\mathfrak{S}_{M}$ on the set of decorated configurations by

$$
\begin{aligned}
\left(s_{k}(d)\right)\left(k^{\prime}\right) & =\left(r\left(s_{k}\left(k^{\prime}\right)\right), c\left(s_{k}\left(k^{\prime}\right)\right),\left(s_{k}(\alpha)\right)\left(k^{\prime}\right)\right), \\
w(d) & =s_{i_{1}}\left(\cdots\left(s_{i_{l}}(d)\right) \cdots\right) \text { for } w=s_{i_{1}} \cdots s_{i_{l}} .
\end{aligned}
$$

Here if $c(k)=-c(k+1)= \pm 1$ then

$$
\left(s_{k}(\alpha)\right)(k)=\alpha(k) \pm 1, \quad\left(s_{k}(\alpha)\right)(k+1)=\alpha(k+1) \pm 1,
$$

and $\left(s_{k}(\alpha)\right)\left(k^{\prime}\right)=\alpha\left(k^{\prime}\right)$ in all other cases.

Set

$$
\begin{aligned}
& T_{k}(d)=\frac{1}{\sqrt{D}} \sum_{a} \sigma_{r(k) r(k+1)}^{\alpha(k)}(a) x_{k}^{a}(d) \\
& \quad \text { for } c(k)=c(k+1)=1, \\
&=\frac{1}{\sqrt{D}} \sum_{a} \sigma_{r(k) r(k+1)}^{\alpha(k)+1}(a) x_{k}^{-a}(d) \\
&=\frac{1}{\sqrt{D}} \sum_{a} \phi_{r(k) r(k+1)}^{\alpha(k)+1}(a) x_{k}^{a}(d) \\
& \quad \text { for } c(k)=c(k+1)=-1, \\
& \frac{1}{\sqrt{D}} \sum_{a} \phi_{r(k) r(k+1)}^{\alpha(k)}(a) x_{k}^{\check{a}}(d) \\
& \quad \text { for } c(k)=-1, c(k+1)=1 .
\end{aligned}
$$

THEOREM 4.1. For any decorated configuration $d$,

(1) $T_{k}\left(s_{k}(d)\right) T_{k}(d)=$ scalar $(1 \leq k<M)$,

$$
\begin{aligned}
& T_{k}\left(s_{k+1} s_{k}(d)\right) T_{k+1}\left(s_{k}(d)\right) T_{k}(d) \\
& \quad=(\text { scalar }) T_{k+1}\left(s_{k} s_{k+1}(d)\right) T_{k}\left(s_{k+1}(d)\right) \\
& \quad(1 \leq k<M-1) .
\end{aligned}
$$

Consider the special case where $\lambda_{i}=\lambda, \mu_{i}=\mu$ and hence $u_{i}=u$, $v_{i}=v$ are independent of $i$ (we call this the trigonometric case). Then $\sigma_{r(k) r(k+1)}^{\alpha}(a)$ depends only on $z=u(k) v(k+1) / u(k+1) v(k)$ and $a$, and $T_{k}(d)$ only on $z$ and $c$. We set

$$
\sigma_{z}(a)=\sigma_{r(k) r(k+1)}^{\alpha}(a), \quad T_{k}(c ; z)=T_{k}(d) .
$$


The recursion relation (4.1) simplifies to

$$
\frac{\sigma_{z}\left(a+\nu_{i}\right)}{\sigma_{z}(a)}=\frac{z q^{a_{t-1 i}}-q^{-a_{t-1 i}}}{z q^{a_{t i+1}+1}-q^{-a_{i+1}-1}} .
$$

Corollary 4.2.

$$
\begin{aligned}
& T_{k}\left(s_{k+1} s_{k}(c) ; z\right) T_{k+1}\left(s_{k}(c) ; z z^{\prime}\right) T_{k}\left(c ; z^{\prime}\right) \\
& \quad=T_{k+1}\left(s_{k} s_{k+1}(c) ; z^{\prime}\right) T_{k}\left(s_{k+1}(c) ; z z^{\prime}\right) T_{k+1}(c ; z) .
\end{aligned}
$$

In the limit $z^{ \pm 1} \rightarrow 0, T_{k}(c ; z)$ tends to $T_{k}(c)^{ \pm 1}$ in $\S 3$.

4.2. Intertwining relations. Proof of Theorem 4.1 is based on an intertwining property of the $T_{k}(d)$, which we shall explain. Fix a decorated configuration $d$. For each $k$, denoting $i+\alpha(k)$ by $j$, we set

$$
\begin{aligned}
a_{k}^{i} & =q \frac{v(k)_{i-1}}{u(k)_{j}}, \quad b_{k}^{i}=\frac{u(k)_{j}}{v(k)_{i}}, \quad \text { for } c(k)=+1, \\
a_{k}^{i} & =-q \frac{v(k)_{i}}{u(k)_{j}}, \quad b_{k}^{i}=-\frac{u(k)_{j}}{v(k)_{i-1}}, \quad \text { for } c(k)=-1, \\
f_{k}^{i} & =\frac{\kappa_{j-1 i-1}}{\kappa_{j i}} \text { for } c(k)=c(k+1)=1, \\
& =\frac{\kappa_{j+1 i}}{\kappa_{j i-1}} \text { for } c(k)=c(k+1)=-1, \\
& =1 \quad \text { otherwise. }
\end{aligned}
$$

Using these, we define $L$-operators for the decorated configuration $d$ by

$$
\mathscr{L}^{i}=\sum_{k=1}^{M-1} l_{1}^{i} \cdots l_{k}^{i}, \quad l_{k}^{i}=a_{k}^{i} b_{k+1}^{i} f_{k}^{i} x_{k}^{\nu_{i}} .
$$

Now exhibiting the $d$ dependence of $l_{k}^{i}, \mathscr{L}^{i}$, we have

\section{Proposition 4.3.}

$$
T_{k}(d) \mathscr{L}^{i}(d)=\mathscr{L}^{i}\left(s_{k}(d)\right) T_{k}(d) \text { for all } i \text { and } 2 \leq k<M .
$$

Proof. First we shall show that it is sufficient to check the following equations,

$$
\begin{aligned}
& T_{k}(d)\left(l_{k-1}^{i}(d)+l_{k-1}^{i}(d) l_{k}^{i}(d)\right) \\
& \quad=\left(l_{k-1}^{i}\left(s_{k}(d)\right)+l_{k-1}^{i}\left(s_{k}(d)\right) l_{k}^{i}\left(s_{k}(d)\right)\right) T_{k}(d) .
\end{aligned}
$$


It follows from (2.2) that

$$
\left[x_{k}^{a}(d), x_{k-1}^{\nu_{i}}(d) x_{k}^{\nu_{i}}(d) x_{k+1}^{\nu_{i}}(d)\right]=0 .
$$

From (2.2), (2.4), (2.5), (4.4), (4.5) and the above relations, we obtain

$$
\begin{gathered}
{\left[T_{k}(d), l_{k^{\prime}}^{i}(d)\right]=0, \quad l_{k^{\prime}}^{i}(d)=l_{k^{\prime}}^{i}\left(s_{k}(d)\right) \quad\left(k^{\prime} \neq k, \quad k \pm 1\right),} \\
{\left[T_{k}(d), l_{k-1}^{i}(d) l_{k}^{i}(d) l_{k+1}^{i}(d)\right]=0} \\
l_{k-1}^{i}(d) l_{k}^{i}(d) l_{k+1}^{i}(d)=l_{k-1}^{i}\left(s_{k}(d)\right) l_{k}^{i}\left(s_{k}(d)\right) l_{k+1}^{i}\left(s_{k}(d)\right) .
\end{gathered}
$$

Therefore the proof reduces to (4.6). We are going to show that (4.6) is equivalent to (4.1). Suppose, for example, $c(k)=-1, c(k+1)=1$ and $\alpha(k)=\alpha(k+1)=j-i$. In this case, $\alpha(k-1)=\alpha(k)$ for $c(k-1)=1,=\alpha(k)-1$ for $c(k-1)=-1$. Then using the notations

$$
\begin{gathered}
T_{k}=T_{k}(d), \quad x_{k^{\prime}}^{a}=x_{k^{\prime}}^{a}(d) \quad\left(k^{\prime}=k-1, k\right), \\
y_{k-1}^{a}=x_{k-1}^{a} x_{k}^{(a+\check{a}) / 2} \text { and } y_{k}^{a}=x_{k}^{-\check{a}},
\end{gathered}
$$

we have

$$
\begin{aligned}
T_{k} \kappa_{j i} & \left(q^{-1} u(k)_{j} v(k+1)_{i} x_{k-1}^{\nu_{i}}-u(k+1)_{j} v(k)_{i} x_{k-1}^{\nu_{i}} x_{k}^{\nu_{i}}\right) \\
= & \left(u(k)_{j-1} v(k+1)_{i-1} y_{k-1}^{\nu_{i}} y_{k}^{\nu_{l}}-q^{-1} u(k+1)_{j-1} v(k)_{i-1} y_{k-1}^{\nu_{i}}\right) \\
& \quad \times \kappa_{j-1 i-1} T_{k} .
\end{aligned}
$$

Then by substituting the expression (4.2) and using the definition of $\phi_{r \tilde{r}}^{\alpha}(a)$, we obtain the recurrence relation (4.1).

LEMMA 4.4. Let $d$ be a decorated configuration, $\mathscr{A}_{k}(d)$ the subalgebra generated by $x_{l}^{a}(d) \quad(a \in L) \quad(k \leq l<M)$ and set

$$
G^{i}(z)=x_{k}^{\nu_{i}}(d)+z x_{k}^{\nu_{i}}(d) g^{\nu_{i}}
$$

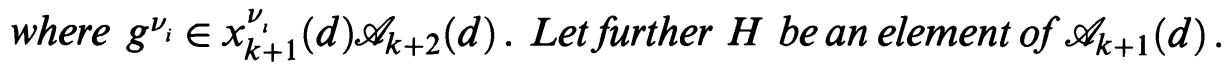
Then for generic $z$ the solution of the following equations

$$
\left[G^{i}(z), H\right]=0, \quad \text { for all } i,
$$

must satisfy

$$
H \in \mathscr{A}_{k+2}(d) \text { and }\left[g^{\nu_{i}}, H\right]=0 .
$$


Proof. Clearly $H \in \mathscr{A}_{k+2}(d)$ satisfying $\left[g^{\nu_{\imath}}, H\right]=0$ is a solution of (4.7). We must show that these are the only solutions for generic $z$. The coefficients of the linear equations (4.7) are polynomials in $z$. Therefore by the specialization argument it is sufficient to prove the assertion for $H(z) \in \mathscr{A}_{k+1}[z]$. Set $\bar{G}^{i}(z)=\left(1-z g^{\nu_{\imath}}\right) x_{k}^{-\nu}(d)$. From $G^{i}(z) \bar{G}^{i}(z)=1+O\left(z^{2}\right)$, it follows that $\left[\bar{G}^{i}(z), H(z)\right]=O\left(z^{2}\right)$. Therefore we have

$$
\begin{aligned}
& {[K, H(z)]=O\left(z^{2}\right),} \\
& K=G^{i}(z) G^{i \mp 1}(z) \bar{G}^{i}(z) \bar{G}^{i \mp 1}(z) \\
& \quad=q^{\kappa}\left(1-z\left(1-q^{-2}\right) g^{\nu_{i}}+O\left(z^{2}\right)\right) \quad \text { for } c(k+1)= \pm 1,
\end{aligned}
$$

with some integer $\kappa$. From this, we obtain

$$
\left[g^{\nu_{\imath}}, H(z)\right]=O(z) .
$$

Putting $z=0$ we have $H(0) \in \mathscr{A}_{k+2}(d)$ and $\left[H(0), g^{\nu_{i}}\right]=0$. Note that this means in particular $\left[G^{i}(z), H(0)\right]=0$. Therefore we can apply the same argument to $(H(z)-H(0)) / z$. By repeating this, the lemma can be proved.

Corollary 4.5. Let $H$ be an element of $\mathscr{A}_{2}(d)$. Then for generic parameters $r(k)(1 \leq k \leq M), \lambda_{i}$ and $\mu_{i}$, the equations

$$
\left[H, \mathscr{L}^{i}(d)\right]=0, \text { for all } i
$$

imply $H \in \mathbf{C} 1$.

Proof. This can be shown by specialization to the trigonometric case. In this case,

$$
\mathscr{L}^{i}(d)=\sum_{k=1}^{M-1} z^{(k)} x_{1}^{\nu_{i}}(d) \cdots x_{k}^{\nu_{i}}(d),
$$

where $z^{(k)}= \pm q^{m^{(k)}} u(k+1) v(1) / v(k+1) u(1)$ for some integer $m^{(k)}$. Then the repeated use of Lemma 4.4 proves the corollary.

Proof of Theorem 4.1. Let

$$
\begin{aligned}
P_{k} & =T_{k}\left(s_{k}(d)\right) T_{k}(d) \\
Q_{k}^{L} & =T_{k}\left(s_{k+1} s_{k}(d)\right) T_{k+1}\left(s_{k}(d)\right) T_{k}(d) \\
Q_{k}^{R} & =T_{k+1}\left(s_{k} s_{k+1}(d)\right) T_{k}\left(s_{k+1}(d)\right) T_{k+1}(d) .
\end{aligned}
$$


From Proposition 4.3, we have

$$
\begin{gathered}
{\left[P_{k}, \mathscr{L}^{i}(d)\right]=0 \text { for } 2 \leq k<M} \\
Q_{k} \mathscr{L}^{i}(d)=\mathscr{L}^{i}\left(s_{k} s_{k+1} s_{k}(d)\right) Q_{k} \\
\text { for } Q_{k}=Q_{k}^{L}, Q_{k}^{R} \text { and } 2 \leq k<M-1 .
\end{gathered}
$$

From Corollary 4.5, we conclude that $P_{k}=$ scalar and $Q_{k}^{L}=$ (scalar) $\times$ $Q_{k}^{R}$. The assertion holds also for the case $k=1$ since only the relations (2.2) are necessary for the proof. In the trigonometric case, comparing the determinant (in some representation) of both sides of (4.3) we see that the scalar factor is 1.

REMARK. The relation between the operators $T$ and the generalized chiral Potts model is as follows. Let $W^{(0)}, X_{i}$ and $Z_{i}(0 \leq i<n)$ be the ones defined in $\S 2.4$. Let us set $M=4$ and consider the decorated configuration $d$ such that

$$
\begin{array}{ll}
d(1)=\left(r^{\prime}, 1,0\right), & d(2)=\left(r^{\dagger},-1,0\right), \\
d(3)=\left(\tilde{r}^{\prime}, 1,0\right), & d(4)=\left(\tilde{r}^{\dagger},-1,0\right) .
\end{array}
$$

On $W^{0} \otimes W^{(0)}$, the algebra $\mathscr{A}(d)$ is realized as follows,

$$
\begin{gathered}
\pi\left(x_{1}^{\nu_{l}}\right)(d)=Z_{i}^{2} \otimes 1, \quad \pi\left(x_{2}^{\nu_{i}}\right)(d)=X_{i}^{-1} X_{i+1} \otimes X_{i} X_{i+1}^{-1}, \\
\pi\left(x_{3}^{\nu_{l}}\right)(d)=1 \otimes Z_{i}^{2} .
\end{gathered}
$$

Then the image of $T_{2}\left(s_{1} s_{3} s_{2}(d)\right) T_{1}\left(s_{3} s_{2}(d)\right) T_{3}\left(s_{2}(d)\right) T_{2}(d)$ under $\pi$ coincides term by term with the $R$-matrix of the generalized chiral Potts model

$$
R\left(\left(r, r^{\prime}\right),\left(\tilde{r}, \tilde{r}^{\prime}\right)\right)=S_{\tilde{r} r^{\prime}}^{-1} T_{r^{\prime} \tilde{r}^{\prime}} \bar{T}_{r \tilde{r}} S_{r \tilde{r}^{\prime}}
$$

Using the Yang-Baxter equation for $T_{k}(d)$, we can prove the YangBaxter equation for the above $R$-matrix.

REMARK. Let us consider the same representation as in the Remark in $\S 3$. Then the matrix elements of $T_{k}(d)$ are given as follows. Set

$$
\begin{aligned}
\omega(a, b, e, f) & =q^{B(f-b, \hat{a}+e) / 2+A(b, f)}, \\
\xi_{r \tilde{r}}^{\alpha}(a, b, e, f) & =\frac{1}{\sqrt{D}} \sigma_{r \tilde{r}}^{\alpha}(f-b) \omega(a, b, e, f), \\
\zeta_{r \tilde{r}}^{\alpha}(a, b, e, f) & =\frac{1}{\sqrt{D}} \phi_{r \tilde{r}}^{\alpha}(g) / \omega(f, a, b, e),
\end{aligned}
$$


where $a, b, e, f \in L$ and $g=(a+e+\hat{a}+\hat{e}) / 2-\hat{f}-b$. Then

$$
\begin{aligned}
& T_{k}(d)_{a_{1}, \ldots, a_{M-1}}^{a_{1}^{\prime}, \ldots, a_{M-1}^{\prime}}=\left(\prod_{l \neq k} \delta_{a_{l} a_{l}^{\prime}}\right) \xi_{r(k) r(k+1)}^{\alpha(k)}\left(a_{k-1}, a_{k}, a_{k+1}, a_{k}^{\prime}\right) \\
& \text { for } c(k)=c(k+1)=1, \\
&=\left(\prod_{l \neq k} \delta_{a_{l} a_{l}^{\prime}}\right) \xi_{r(k) r(k+1)}^{\alpha(k)+1}\left(a_{k+1}, a_{k}^{\prime}, a_{k-1}, a_{k}\right) \\
&=\left(\prod_{l \neq k} \delta_{a_{l} a_{l}^{\prime}}\right) \zeta_{r(k) r(k+1)}^{\alpha(k)+1}\left(a_{k-1}, a_{k}, a_{k+1}, a_{k}^{\prime}\right) \\
& \zeta_{r(k) r(k+1)}^{\alpha(k)}\left(a_{k+1}, a_{k}^{\prime}, a_{k-1}, a_{k}\right) \text { for } c(k)=1, c(k+1)=-1, \\
& \text { for } c(k)=-1, c(k+1)=1 .
\end{aligned}
$$

$\xi_{\mathrm{r} \widetilde{\mathrm{r}}}^{\alpha}(\mathrm{a}, \mathrm{b}, \mathrm{e}, \mathrm{f})=$
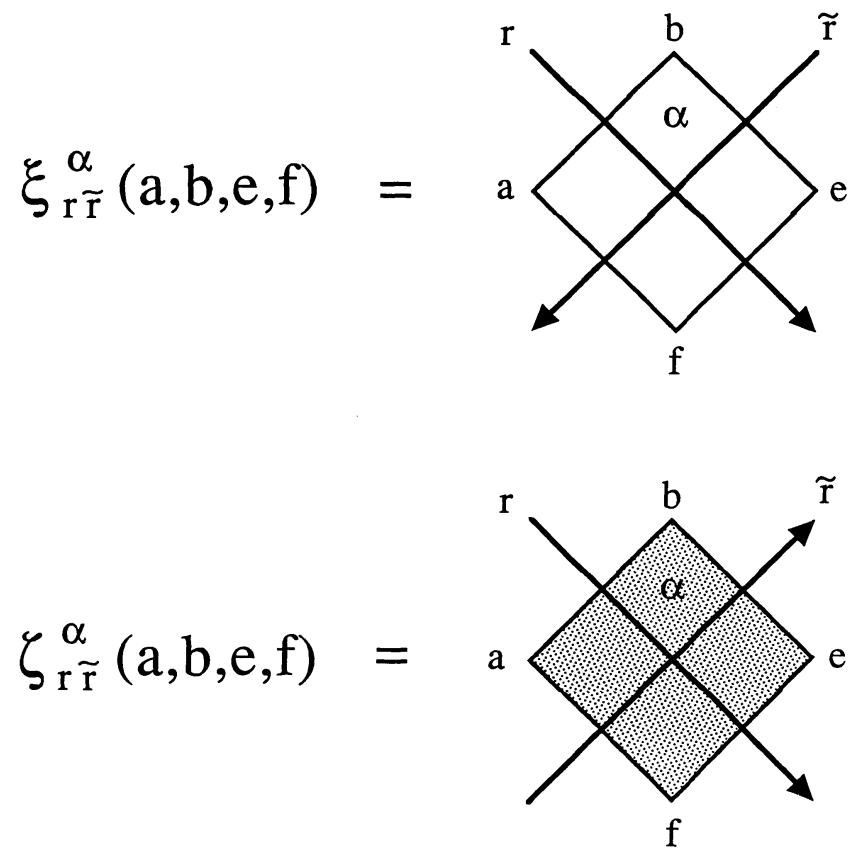

FIGURE 4.2

Matrix elements of $T_{k}(d)$ 
In terms of $\xi$ and $\zeta$, the Yang-Baxter equations become

$$
\begin{aligned}
& \sum_{g} \xi_{r r^{\prime \prime}}^{\alpha}(a, b, g, f) \xi_{r^{\prime} r^{\prime \prime}}^{\alpha-1}(b, c, d, g) \xi_{r r^{\prime}}^{\alpha-1}(f, g, d, e) \\
& \quad=(\text { scalar }) \sum_{g} \xi_{r r^{\prime}}^{\alpha}(a, b, c, g) \xi_{r r^{\prime \prime}}^{\alpha-1}(g, c, d, e) \xi_{r^{\prime} r^{\prime \prime}}^{\alpha}(a, g, e, f), \\
& \sum_{g} \zeta_{r r^{\prime \prime}}^{\alpha}(a, b, g, f) \zeta_{r^{\prime} r^{\prime \prime}}^{\alpha-1}(b, c, d, g) \xi_{r r^{\prime}}^{\alpha}(f, g, d, e) \\
& \quad=(\text { scalar }) \sum_{g} \xi_{r r^{\prime}}^{\alpha-1}(a, b, c, g) \zeta_{r r^{\prime \prime}}^{\alpha-1}(g, c, d, e) \zeta_{r^{\prime} r^{\prime \prime}}^{\alpha}(a, g, e, f), \\
& \sum_{g} \zeta_{r r^{\prime \prime}}^{\alpha}(g, f, a, b) \xi_{r^{\prime} r^{\prime \prime}}^{\alpha}(b, c, d, g) \zeta_{r r^{\prime}}^{\alpha-1}(d, e, f, g) \\
& \quad=(\text { scalar }) \sum_{g} \zeta_{r r^{\prime}}^{\alpha}(c, g, a, b) \zeta_{r r^{\prime \prime}}^{\alpha-1}(d, e, g, c) \xi_{r^{\prime} r^{\prime \prime}}^{\alpha-1}(a, g, e, f),
\end{aligned}
$$

where $a, b, c, d, e, f, g \in L$ and $r, r^{\prime}, r^{\prime \prime} \in \mathscr{C}_{\gamma}$. Note that these equations are equivalent

4.3. Yang-Baxterization for general $B$. We now return to the case of general bilinear form $B$. Consider the operators $T_{k}(c)$ with $c_{j}=$ $1(\forall j)$ which satisfies the ordinary braid relation. Following [14] we say that a set of polynomials $\left\{T_{k}(c ; z)\right\} \subset \mathscr{A}(c)[z]$ is a YangBaxterization of $\left\{T_{k}(c)\right\}$ if $T_{k}(c ; 0)=T_{k}(c)$ and the Yang-Baxter equation (4.3) holds. Clearly if $\left\{T_{k}(c ; z)\right\}$ is a Yang-Baxterization, then so is $\left\{T_{k}^{\prime}(c ; z)\right\}$ given by $T_{k}^{\prime}(c ; z)=\varphi(z) T_{k}(c ; z)$ with some $\varphi(z) \in \mathbf{C}[z]$. We say that these two Yang-Baxterizations are equivalent. A non-trivial Yang-Baxterization is one which is not equivalent to $\left\{T_{k}(c)\right\}$ itself.

Let $\left\{T_{k}(c ; z)\right\}$ be a Yang-Baxterization, and define $\phi(a)$ and its Fourier transform $\tilde{\phi}(a)$ by

$$
\begin{aligned}
T_{k}(c ; z) & =\sum_{a} f(a)^{*}\left(1+z \phi(a)+O\left(z^{2}\right)\right) x_{k}^{a}(c), \\
\tilde{\phi}(a) & =\frac{1}{D} \sum_{b} \phi(b) q^{B(a, b)} .
\end{aligned}
$$

Choosing an equivalent $T_{k}(c ; z)$ as necessary, we assume $\tilde{\phi}(0)=0$ without loss of generality.

Lemma 4.6. (i) For any $a, b \in L$ we have

(4.9a) $\quad \tilde{\phi}(a)=\tilde{\phi}(\hat{a})$,

(4.9b) $\quad \tilde{\phi}(b)\left(\tilde{\phi}(a)\left(a^{-B(a, b)}-1\right)-\delta_{a b} q^{-B(a, a)}+\delta_{\hat{a} b}\right)=0$.

(ii) If $a \neq b$ and $\tilde{\phi}(a) \tilde{\phi}(b) \neq 0$, then $b=\hat{a}$, or $B(a, b)=0$. 

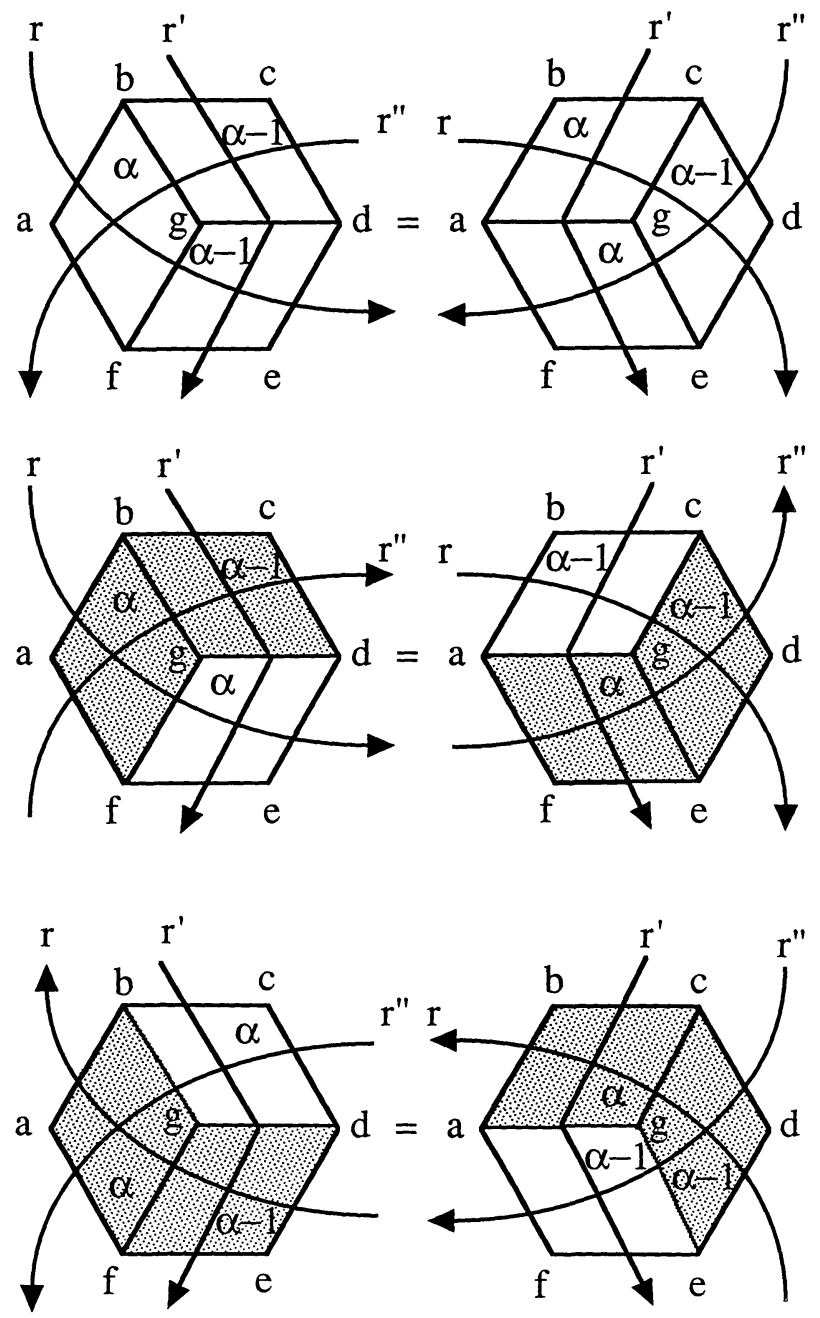

FIGURE 4.3

Yang-Baxter equation

Proof. Picking the coefficients of $z^{\prime}$ and $z z^{\prime}$ in the Yang-Baxter equation (4.3), we find (4.9a) and (4.9b) respectively. (The coefficients of $z$ give trivial identity.) The second assertion follows from (4.9b).

For $a \in L$, define $a^{(j)}$ inductively by $a^{(0)}=a$ and $a^{(j)}=\widehat{a}^{\prime}$ with $a^{\prime}=a^{(j-1)}$.

\section{LemMa 4.7. Assume that}

$$
B(a, a) \neq 0 \text { for } a \neq 0 \text {. }
$$


Suppose $\tilde{\phi}(a) \neq 0$, and let $r$ be the least positive integer such that $a^{(r)}=a$. Then

(i) $a+a^{(1)}+\cdots+a^{(r-1)}=0$,

(ii)

$$
\begin{aligned}
B\left(a^{(i)}, a^{(j)}\right) & =B(a, a) & & \text { if } i \equiv j \bmod r \\
& =-B(a, a) & & \text { if } i+1 \equiv j \bmod r \\
& =0 & & \text { otherwise. }
\end{aligned}
$$

(iii) $a, a^{(1)}, \ldots, a^{(r-2)}$ are linearly independent over $\mathbf{Z} / N \mathbf{Z}$.

Proof. Note that (4.10) implies $\widehat{a} \neq a$ if $a \neq 0$, and hence $r>1$. Assertion (i) follows from this, since under $~$ the left-hand side is invariant. Assertion (ii) is a consequence of Lemma 4.6(ii). To show (iii), suppose there exists a relation $\sum_{j=0}^{r-2} c_{j} a^{(j)}, c_{j} \in \mathbf{Z} / N Z$. Then we have for $0 \leq k \leq r-2$

$$
0=B\left(\sum_{j=0}^{r-2} c_{j} a^{(j)}, a+a^{(1)}+\cdots+a^{(k)}\right)=c_{k} B(a, a)
$$

by (ii). Since $B(a, a) \neq 0$ by (4.10) we have $c_{k}=0$ for all $k$.

Now let $N=p$ be an odd prime. Then $L \simeq \mathbf{F}_{p}^{l}$ where $\mathbf{F}_{p}$ is the field with $p$ elements. Lemma 4.7 means that on the linear subspace $V=\bigoplus_{j=0}^{r-2} \mathbf{F}_{p} a^{(j)}$ the bilinear form $B$ has the form $\lambda B_{r}$, where $\lambda=$ $-B(a, a) / 2$ and $B_{r}$ denotes the bilinear form associated with the $\mathfrak{s l}(r)$ chiral Potts model. Note that $V$ is invariant under - . Set $W=\{b \in L \mid B(b, V)=0\}=\{b \in L \mid B(V, b)=0\}$. For $b \in L$, the element $d=b-\sum_{j=0}^{r-2} c_{j} a^{(j)}$ with $c_{k}=B\left(b, a+a^{(1)}+\cdots+a^{(k)}\right)$ belongs to $W$. From this we see that $L=V \oplus W$. Thus we have

Proposition 4.8. Let $N=p$ be an odd prime, and assume (4.10). Then there exists a non-trivial Yang-Baxterization of $T_{k}(c)$ if and only if $B$ can be written in a suitable basis as a direct sum

$$
B=\lambda B_{r} \oplus B^{\prime},
$$

where $\lambda \in\left(\mathbf{F}_{p}\right)^{\times}, B_{r}$ is the bilinear form of $\mathfrak{s l}(r)$ chiral Potts model, and $B^{\prime}$ is a non-singular bilinear form.

In particular, the case discussed in [14] has a non-trivial YangBaxterization if and only if $s+s^{*}=0, \pm 1$ (in the notation of [14]). 
Acknowledgment. The authors would like to thank I. Cherednik, V. Jones, M. Kashiwara, T. Kohno, H. Murakami, J. Murakami, T. Oda, N. Reshetikhin, E. Witten and D. Zagier for discussions. K. M. is supported in part by the Grant-in-aid for Scientific Research from the Ministry of Education, Science and Culture of Japan No. 01790204. T. M. is partially supported by NSF Grant No. PHY8904035, supplemented by funds from NASA.

\section{REFERENCES}

[1] H. Au-Yang, B. M. McCoy, J. H. H. Perk, S. Tang and M.-L. Yan, Commuting transfer matrices in the chiral Potts models: Solutions of star-triangle equations with genus > 1, Phys. Lett. A, 123 (1987), 219-223.

[2] B. M. McCoy, J. H. H. Perk, S. Tang and C. H. Sah, Commuting transfer matrices for the four-state self-dual chiral Potts model with a genus-three uniformizing Fermat curve, Phys. Lett. A, 125 (1987), 9-14.

[3] H. Au-Yang, B. M. McCoy, J. H. H. Perk and S. Tang, Solvable models in statistical mechanics and Riemann surfaces of genus greater than one, in 'Algebraic Analysis', Eds. M. Kashiwara and T. Kawai, 1 (1988), 29-39, Academic.

[4] R. J. Baxter, J. H. H. Perk and H. Au-Yang, New solutions of the star-triangle relations for the chiral Potts model, Phys. Lett A, 128 (1988), 138-142.

[5] V. V. Bazhanov and Yu. G. Stroganov, Chiral Potts models as a descendant of the six-vertex models, J. Stat. Phys., 59 (1990), 799-817.

[6] V. V. Bazhanov and R. M. Kashaev, Cyclic L-operators related with 3-state R-matrix, Comm. Math. Phys., 136 (1991), 607-623.

[7] E. Date, M. Jimbo, K. Miki and T. Miwa, R-matrix for cyclic representations of $U_{q}(\widehat{\mathfrak{s l}}(3, \mathbf{C}))$ at $q^{3}=1$, Phys. Lett A, 148 (1990), 45-49.

[8] _ New $R$-matrices associated with cyclic representations of $U_{q}\left(A_{2}^{(2)}\right)$, to appear in Publ. RIMS, Kyoto Univ., 27 (1991).

[9] R. M. Kashaev and V. V. Mangazeev, The four-state solution of the Yang-Baxter equation, Phys. Lett. A, 150 (1990), 375-379.

[10] V. V. Bazhanov, R. M. Kashaev, V. V. Mangazeev and Yu. G. Stroganov, $\left(Z_{N} \times\right)^{n-1}$ generalization of the chiral Potts model, Comm. Math. Phys., 138 (1991), 393-408.

[11] E. Date, M. Jimbo, K. Miki and T. Miwa, Generalized chiral Potts models and minimal cyclic representations of $U_{q}(\widehat{\mathfrak{g l}}(n, \mathbf{C}))$, Comm. Math. Phys., 137 (1991), 133-147.

[12] V. A. Fateev and A. B. Zamolodchikov, Self-dual solutions of the star-triangle relations in $\mathbf{Z}_{N}$ models, Phys. Lett. A, 92 (1982), 37-39.

[13] T. Kobayashi, H. Murakami and J. Murakami, Cyclotomic invariants for links, Proc. Japan Acad., 64A (1988), 235-238.

[14] V. F. R. Jones, Baxterization, in Yang-Baxter Equations, Conformal Invariance and Integrability in Statistical Mechanics and Field Theory, Eds. M.N. Barber and P. A. Pearce, World Scientific, Singapore 1990, 1-13.

[15] D. N. Yetter, Markov algebras, Contemporary Math., 78 (1988), 705-730.

[16] G. Burde and H. Zieschang, Knots, de Gruyter, Berlin, 1985.

[17] D. Goldschmidt and V. F. R. Jones, Metaplectic link invariants, Geometriae Dedicata, 31 (1989), 165-191. 
[18] J. Levine, An algebraic classification of some knots of codimension two, Comm. Math. Helv., 45 (1970), 185-198.

[19] H. F. Trotter, On $S$ equivalence of Seifert matrices, Inventiones Math., 20 (1973), 173-207.

Received December 13, 1990.

OSAKA UNIVERSITY

TOYONAKA, Osaka 560, JAPAN

KYOTO UNIVERSITY

KYOTO 606, JAPAN

Yukawa Institute for Theoretical Physics

KYOTO UNIVERSITY

KYOTO 606, JAPAN

ReseARCh INSTITUTE fOR MAThematical ScIENCES

KYOTO UNIVERSITY

KYOTO 606, JAPAN

AND

ITP

UNIVERSity of CALIFornia, SANTA Barbara

SANTA BARBARA, CA 93106 


\section{PACIFIC JOURNAL OF MATHEMATICS EDITORS}

V. S. VARAdARAJAN

(Managing Editor)

University of California

Los Angeles, CA 90024-1555

Herbert Clemens

University of Utah

Salt Lake City, UT 84112

F. Michael CHRIST

University of California

Los Angeles, CA 90024-1555

ThOMAs ENRIGHT

University of California, San Diego

La Jolla, CA 92093
Nicholas ERCOLANI

University of Arizona

Tucson, AZ 85721

R. FINN

Stanford University

Stanford, CA 94305

VAUGHAN F. R. JoNES

University of California

Berkeley, CA 94720

SteVen KercKhofF

Stanford University

Stanford, CA 94305
C. C. MOORE

University of California

Berkeley, CA 94720

MARTIN SCHARLEMANN

University of California

Santa Barbara, CA 93106

HAROLD STARK

University of California, San Diego

La Jolla, CA 92093

\section{ASSOCIATE EDITORS}
R. Arens
E. F. BeCKenBACH
B. H. NeumanN
F. WolF
(1904-1989)
K. YoshidA (1906-1982)

TIONS

UNIVERSITY OF ARIZONA

UNIVERSITY OF BRITISH COLUMBIA

UNIVERSITY OF OREGON

UNIVERSITY OF SOUTHERN CALIFORNIA

CALIFORNIA INSTITUTE OF TECHNOLOGY

UNIVERSITY OF CALIFORNIA

MONTANA STATE UNIVERSITY

STANFORD UNIVERSITY

UNIVERSITY OF HAWAII

UNIVERSITY OF NEVADA, RENO

UNIVERSITY OF TOKYO

NEW MEXICO STATE UNIVERSITY

UNIVERSITY OF UTAH

OREGON STATE UNIVERSITY

WASHINGTON STATE UNIVERSITY

UNIVERSITY OF WASHINGTON 


\section{Pacific Journal of Mathematics}

Vol. 154, No. $1 \quad$ May, 1992

Richard Arens, Pseudo regular elements in a normed ring $\ldots \ldots \ldots \ldots \ldots 1$

Joan Birman and William W. Menasco, Studying links via closed braids.

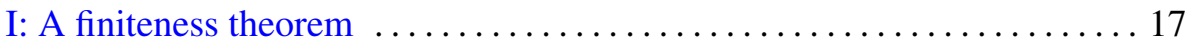

Etsurō Date, Michio Jimbo, Kei Miki and Tetsuji Miwa, Braid group

representations arising from the generalized chiral Potts models ....... 37

Toshihiro Hamachi, A measure theoretical proof of the Connes-Woods

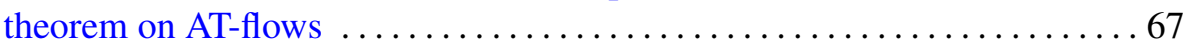

Allen E. Hatcher and Ulrich Oertel, Affine lamination spaces for surfaces ....................................... 87

David Joyner, Simple local trace formulas for unramified $p$-adic groups $\ldots .103$

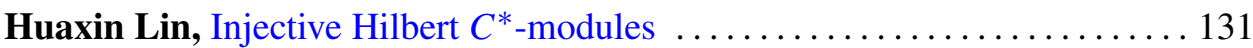

John Marafino, The boundary of a simply connected domain at an inner

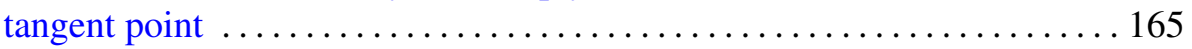

Gonzalo Riera and Rubi Rodriguez, The period matrix of Bring's curve . . 179 